\title{
Induced Drag Characteristics Based on Wake Surface Integration for Wing-In-Ground Effect
}

\author{
By Seung-Yun SHIN, ${ }^{1)}$ Kyung-Hyun WHANG, ${ }^{2)}$ Kyun-Seok KIM ${ }^{3)}$ and Jang-Hyuk KwoN ${ }^{3)}$ \\ ${ }^{1)}$ Korean Air Research and Development Center, Daejeon, Korea \\ ${ }^{2)}$ Korean Institute of Machinery and Material, Daejeon, Korea \\ ${ }^{3)}$ Department of Aerospace Engineering, Korea Advanced Institute of Science and Technology, Daejeon, Korea
}

(Received April 14th, 2009)

\begin{abstract}
The present paper predicts the induced drag in the ground effect using wake surface integration. The total drag can be decomposed into physical drag components such as wave, profile, and induced drag. First, we confirmed that applying wake surface integration to cases of ground effect is theoretically valid as long as a moving boundary condition is implemented for the ground surface. Second, total drag comparison between body surface integration and wake surface integration is performed to numerically identify the disparity. Last, the induced drag component is extracted at various heights. In addition, endplates are mounted on wing tips to determine their impact on the ground effect. This study uses the Navier-Stokes solver for aerodynamic analysis, and a Clark-Y wing with DHMTU concept is generated. As a result, the wake surface integration satisfactorily shows the characteristics of the induced drag in the ground effect with the Clark-Y wing and wing-endplate combinations.
\end{abstract}

Key Words: Induced Drag, Wake Integration, Ground Effect, WIG, CFD-Based Drag Prediction

\section{Nomenclature}

$b$ : wing span length

$\bar{c}$ : wing mean geometric chord length

$C_{\mathrm{D}}:$ drag coefficient

$D$ : total drag force

$h$ : stagnation enthalpy

$H$ : height

$M$ : Mach number

$n$ : surface normal vector

$R e$ : Reynolds number

$R$ : gas constant

$S$ : boundary surface

$V:$ velocity vector $=(u, v, w)$

$X, Y, Z$ : Cartesian coordinate system

$\tau$ : viscous stress tensor

$\rho$ : fluid density

$\Delta$ : perturbation

$\psi$ : cross-flow stream function

$\xi$ : stream-wise vorticity

Subscripts
p: pressure
f: frictional
i: induced
s: entropy
$\infty$ : free stream

\section{Introduction}

The WIG (Wing-In-Ground effect vehicle) is expected to be used in promising new types of transportation. The WIG

(C) 2010 The Japan Society for Aeronautical and Space Sciences takes advantage of the ground effect near a ground or water surface. The ground effect has been known since the start of manned flight. Early WIGs were developed concurrently in both the former USSR and Germany for military purposes. However, mature WIGs have not been developed because of the classified nature of research on the ground effect. In addition, practical use of this technology involves several technical problems that are mainly related to longitudinal stability and size reduction.

WIG aerodynamic concepts include two major phenomena. First, the lift coefficient varies with airfoil shape. Generally, it increases near the ground because of the ram effect. However, it can sometimes decrease because of the Venturi effect. In addition, wing tip vortices in free flight are weakened near the ground because the space restriction prevents vortices forming completely. This, in turn, increases the effective aspect ratio (AR). As a result of these factors, the lift-to-drag (L/D) ratio generally increases, and the efficiency of the WIG is superior to that of a conventional aircraft. ${ }^{1)}$

Body surface integration of pressure and shear stress also called the near-field method, is most commonly used to predict CFD-based drag. However, body surface integration has difficulties in accurately predicting skin friction, especially on a complex geometry, because it requires geometrical approximation of a curved surface using flat facets. Therefore, many researchers have attempted to apply wake surface integration, or far-field integration, based on the momentum conservation theorem for CFD computations. ${ }^{2)}$

Yu et al. ${ }^{3)}$ and Lock ${ }^{4)}$ first attempted to use wake surface integration for predicting drag. This method has been successful for predicting lift, induced drag and wave drag using 
the Euler equation. ${ }^{5,6)}$ Profile drag has been also calculated by solving the Reynolds-averaged Navier-Stokes equation. ${ }^{7,8)}$ Another advantage of using wake integration for estimation is that the total drag component can be decomposed into the wave, profile, and induced drag by integrating the wake surface $;^{9)}$ this is the main reason why we used wake integration to estimate induced drag in this study.

Flow-field or mid-field integration, a recently proposed approach, can compute drag components from volume integration. This approach can isolate the spurious drag component attributable to numerical diffusion, enabling more accurate drag prediction. ${ }^{10-12)}$

This study predicts the induced drag in ground effect is using wake surface integration. First, we confirmed whether or not application of wake surface integration to cases of the ground effect is valid when a moving boundary condition is implemented for the ground surface.

In ground-effect experiments, a moving belt or towing carriage is often used to simulate real flight near the ground. Even there is a fixed wall; the boundary layer on the ground should be controlled using suction or blowing devices. Therefore, it is reasonable that a moving boundary condition is implemented on a ground surface for CFD calculation. The characteristics of a wing for ground effect in two ground conditions, fixed and moving ground, have been clearly shown by Barber ${ }^{13)}$ and Chun et al. ${ }^{14)}$

Moreover, the total drag is compared between body surface integration and wake surface integration to quantify the disparity. Finally, the induced drag component is extracted at various heights. In addition, endplates are mounted on wing tips to determine the impact on the ground effect. This study uses a Navier-Stokes solver for aerodynamic analysis and generated a Clark- $\mathrm{Y}^{15)}$ wing with DHMTU (Department of Hydromechanics of the Marine Technical University) concept. ${ }^{16,17)}$

\section{Drag Prediction Methods}

This section explains the drag prediction methods used in this study. We found that wake surface integration can be used for cases of ground effect by applying a moving boundary condition.

The control volume and coordinate system used for the drag analysis are shown in Fig. 1.

\subsection{Body surface integration (BSI) method}

Usually drag is computed by integrating the body surface for the CFD calculation as follows:

$$
\begin{aligned}
D & =\iint_{S_{\text {body }}}\left(-p n_{x}+\tau_{x x} n_{x}+\tau_{x y} n_{y}+\tau_{x z} n_{z}\right) \mathrm{d} S \\
D_{\mathrm{p}} & =\iint_{S_{\text {body }}}-p n_{x} \mathrm{~d} S, \quad D_{\mathrm{f}}=\iint_{S_{\text {body }}} \tau_{x} \cdot n \mathrm{~d} S
\end{aligned}
$$

$S_{\text {body }}$ denotes the surface of the body. $D_{\mathrm{p}}$ and $D_{\mathrm{f}}$ denote the pressure and skin friction drag, respectively, in Eq. (2).

\subsection{Wake surface integration (WSI) method}

Total drag can be expressed as a surface integral of any control volume enclosing the body by using the integral form

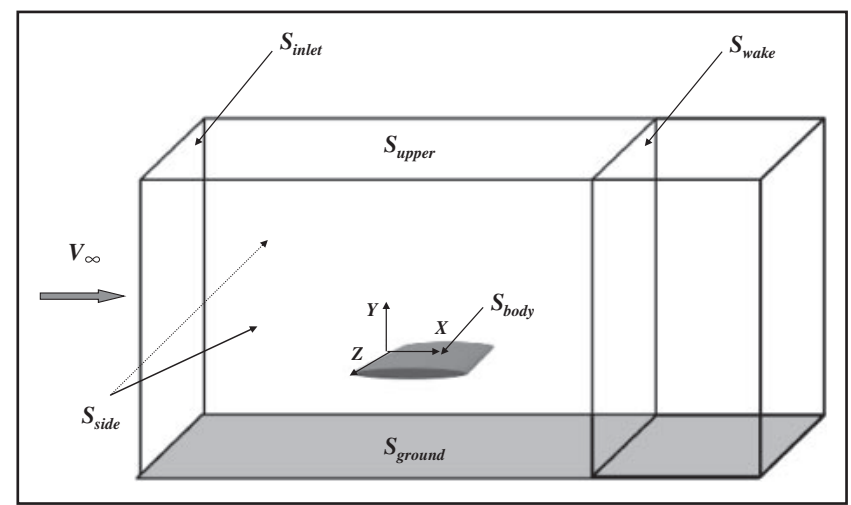

Fig. 1. Control volume for body and wake integration.

of the momentum equations with the following assumptions.

\section{General assumptions:}

(1) The free stream, $V_{\infty}$, is aligned along the $x$-direction and the control volume is considered to be a cuboid aligned with the Cartesian coordinate system.

(2) $S_{\text {upper }}, S_{\text {inlet }}$, and $S_{\text {side }}$ are sufficiently far from the wing so that the flow on these surfaces is undisturbed.

With respect to these assumptions, the following expression is obtained for drag near the ground.

$$
\begin{aligned}
D= & \iint_{S_{\text {wake }}}\left\{\left(p_{\infty}-p\right)-p u\left(u-u_{\infty}\right)+\tau_{x x}\right\} \mathrm{d} S \\
& +\iint_{S_{\text {ground }}} \tau_{x z} \mathrm{~d} S
\end{aligned}
$$

$S_{\text {wake }}$ and $S_{\text {ground }}$ denote the surface of the wake and the ground, respectively.

\section{Proposed assumptions:}

(3) $\tau_{x x}$ of the viscous stress terms in the wake surface is negligible if $S_{\text {wake }}$ is located behind one root chord length in the Cartesian coordinate system. ${ }^{9)}$

(4) $\tau_{x z}$ of the viscous stress terms in the ground surface is also negligible when the moving boundary condition is applied to $S_{\text {ground }}$. Figure 2 shows the velocity profiles on the leading edge $(X=0.0)$ and the mid-point $(X=0.5)$ of the wing mean geometric chord between the wing and the ground to confirm this assumption. Note that velocity gradients remain near 0 . Height ratio (HR) is defined as $H / \bar{c}$. The definition of height is detailed in section 4.3 and Fig. 8.

By applying proposed assumptions (3) and (4), Eq. (3) is transformed into Eq. (4), which is identical to the general form of wake surface integration. This suggests that applying wake surface integration to cases of ground effect is valid as long as a moving boundary condition is implemented for the ground surface. If a fixed boundary condition is applied, drag would increase because of the term $\tau_{x z}$.

$$
D=\iint_{S_{\text {wake }}}\left\{\left(p_{\infty}-p\right)-\rho u\left(u-u_{\infty}\right)\right\} \mathrm{d} S
$$

Equation (4) can be divided into the following three drag components: 


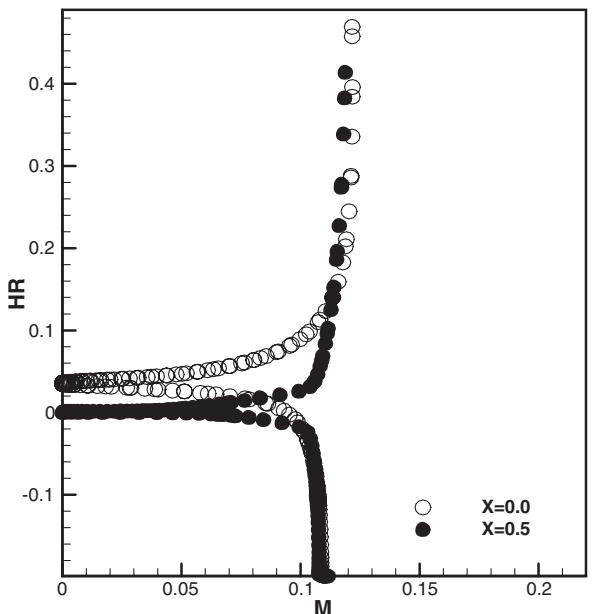

Fig. 2. Velocity profiles between wing and ground.

$$
\begin{aligned}
D \approx & -\rho_{\infty} \iint_{S_{\text {wake }}} \Delta h \mathrm{~d} S+\frac{P_{\infty}}{R} \iint_{S_{\text {wake }}} \Delta s \mathrm{~d} S \\
& +\frac{\rho_{\infty}}{2} \iint_{S_{\text {wake }}} \psi \xi \mathrm{d} S
\end{aligned}
$$

$\Delta h$ in the first term is usually negligible with limited cases such as external power sources switched off, and then, the final expression of the wake surface integration is given by Eq. (6). ${ }^{9)}$

$$
\begin{aligned}
D & =\frac{P_{\infty}}{R} \iint_{S_{\text {wake }}} \Delta S \mathrm{~d} S+\frac{\rho_{\infty}}{2} \iint_{S_{\text {wake }}} \psi \xi \mathrm{d} S \\
D_{\mathrm{s}} & =\frac{P_{\infty}}{R} \iint_{S_{\text {wake }}} \Delta s \mathrm{~d} S, \quad D_{\mathrm{i}}=\frac{\rho_{\infty}}{2} \iint_{S_{\text {wake }}} \psi \xi \mathrm{d} S
\end{aligned}
$$

$D_{\text {s }}$ is called entropy drag; it includes the wave and profile drag components. In the present flow condition, wave drag does not contribute to this term because the cruising velocity is too low to form shock waves. $D_{\mathrm{i}}$ corresponds to the induced drag in Maskell's form.

\section{Induced Drag Correction Method}

This section explains two types of correction methods that improve the consistency of the induced drag.

\subsection{Near-wake correction (NWC)}

There are significant velocity variations in the near wake along the downstream direction. An abrupt drop is observed in the induced drag calculation due to exclusion of a higherorder term. This term can be significant in the very-near wake. For better consistency, the higher-order term should be considered ${ }^{2)}$ and then the corrected induced drag is given by:

$$
\begin{aligned}
D_{\mathrm{i}_{\mathrm{NWC}}}= & \frac{\rho_{\infty}}{2} \iint_{S_{\text {wake }}} \psi \xi \mathrm{d} S \\
& -\frac{\rho_{\infty}}{2}\left(1-M_{\infty}^{2}\right) \iint_{S_{\text {wake }}}(\Delta u)^{2} \mathrm{~d} S
\end{aligned}
$$

Figure 3 shows an improvement in the consistency of the induced drag coefficient near the trailing edge $(X=1.0)$ for both height ratios (HR) 0.1 and 0.7. NWC indicates the

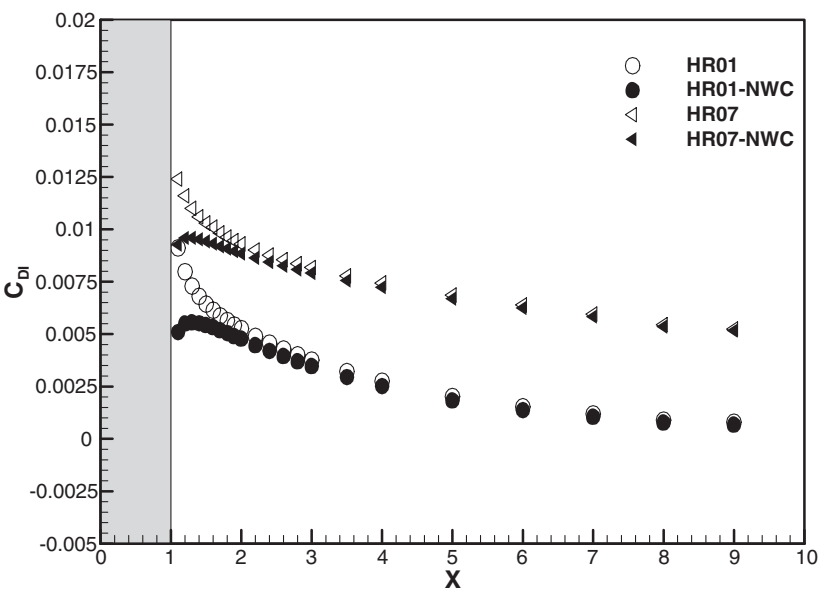

Fig. 3. Near-wake induced drag correction.

induced drag along the downstream direction after the near-field correction.

\subsection{Far-wake correction (FWC)}

The induced drag decays gradually to zero owing to wake diffusion caused by numerical smoothing and the coarse grid at the far downstream, as shown in Fig. 3.

To predict the consecutive induced drag, the amount of wake diffusion must be added to compensate the induced drag loss. The amount of diffusion in the wing tip vortex is found by calculating the increase in the entropy from the wing tip to the wake surface at downstream. The nominal value of the induced drag is chosen by taking the wake position $(X=2.0)$ of one root chord aft of the trailing edge. $^{8)}$

This compensation leads an additional term to correct the induced drag as expressed in Eq. (9). Moreover, a smaller size of cross-flow plane was used to improve the accuracy of the calculation as described by Hunt et al. ${ }^{6)}$

Consequently, the sum of the original and additional two terms is almost constant at arbitrary wake positions. This suggests that the total induced drag is almost conserved in the wake region as indicated by HR01-FWC and HR07FWC in Fig. 4. NWC is the induced drag after the near-field computation; ENT is the entropy drag; and FWC is the induced drag after compensation by the increment in ENT.

$$
\begin{aligned}
D_{\mathrm{i}_{\mathrm{FWC}}} & =D_{\mathrm{i}_{\mathrm{NWC}}}+\frac{p_{\infty}}{R} \iint_{S_{\mathrm{wake}}} \Delta s \mathrm{~d} S \\
\Delta s & \approx-R \ln \frac{p_{T}}{p_{T_{\infty}}}
\end{aligned}
$$

The final expression for induced drag is given by:

$$
\begin{aligned}
D_{\mathrm{i}}= & \frac{\rho_{\infty}}{2} \iint_{S_{\text {wake }}} \psi \xi \mathrm{d} S \\
& -\frac{\rho_{\infty}}{2}\left(1-M_{\infty}{ }^{2}\right) \iint_{S_{\text {wake }}}(\Delta u)^{2} \mathrm{~d} S \\
& +\frac{p_{\infty}}{R} \iint_{S_{\text {wake }}} \Delta s \mathrm{~d} S
\end{aligned}
$$




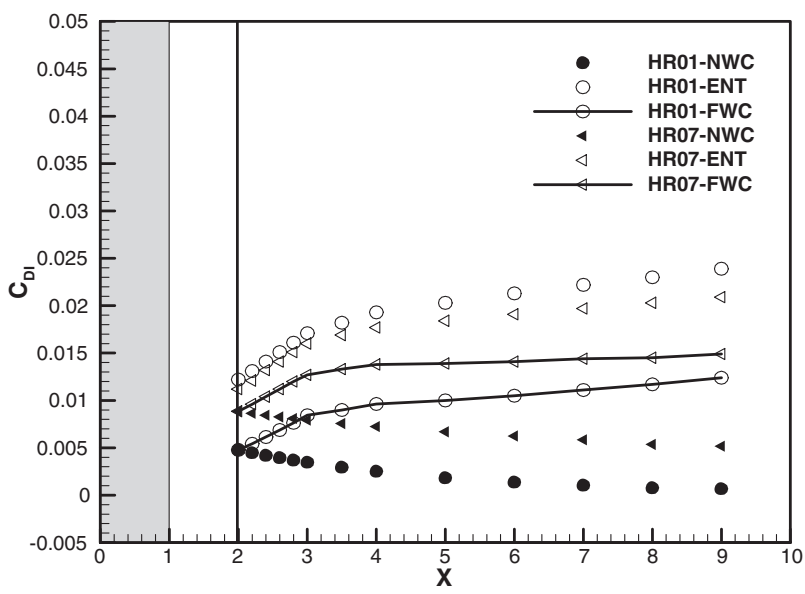

Fig. 4. Far-wake induced drag correction.

\section{Aerodynamic Analysis}

\subsection{Flow solver and flow condition}

This study performed three-dimensional viscous subsonic flow computations at Mach number 0.11 and Reynolds number $2.55 \times 10^{6}$. For these flow computations, Weiss and Smith's preconditioning method was used in the KFLOW code. ${ }^{18)}$ The preconditioning method has already been used for the wake surface integration by Chao and van Dam, ${ }^{8)}$ and the low Mach preconditioning greatly improves the consistency of the drag when wake integration is applied. The preconditioned governing equations are solved by implementing the cell-centered finite volume method in space. The artificial dissipation term of Roe's FDS should include the preconditioning effect to guarantee the accuracy of solution at a low Mach number. In addition, the MUSCL (Monotone Upstream-centered for Conservative Law) scheme is used to obtain the third-order solution accuracy for convection terms. In time, the DADI (Diagonalized Alternate Directional Implicit) scheme is implemented, and the local time stepping method is used with preconditioned velocities and the speed of sound for obtaining a steady-state solution. ${ }^{19)}$ The $k-\omega \mathrm{WD}+$ model $^{20)}$ is used as a turbulent model, and a fully turbulent flow is assumed in this computation.

\subsection{Grid generation and cross-flow plane cutting}

An H-type structured grid was used to generate the grid for an attack angle of $0^{\circ}$ and height ratios of $0.7,0.5,0.3$, 0.2 , and 0.1 ; the wing tip was extended rather than merged to attach the endplate. Unfortunately, there was interference between the endplates and the ground at a height ratio of 0.1 . So this case could not be generated.

The numbers of the grid were 3,595,669 for the wing and $3,978,553$ for the wing with the endplate blocks. The first cell size was $10^{-5}$. The computations were conducted with 9 and 18 CPUs, respectively.

The nodes on the cutting plane in Fig. 6 were regenerated by the intersection of the original three-dimensional grid in Fig. 5, and all flow variables were defined at the new nodes by linear interpolation.

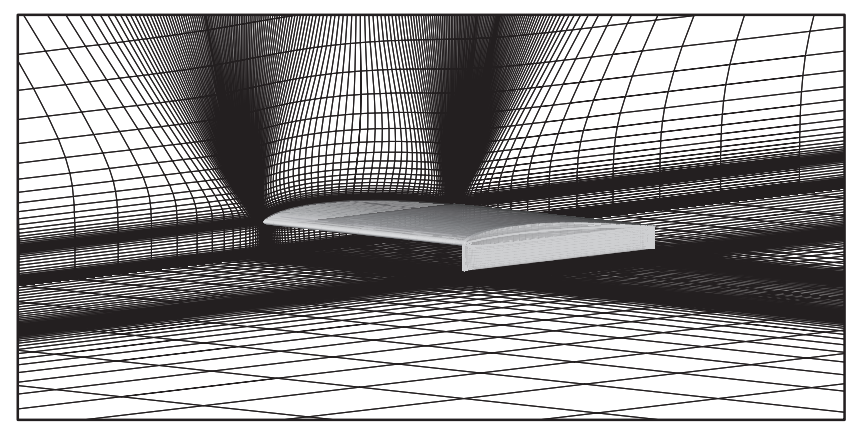

Fig. 5. H-type grid.

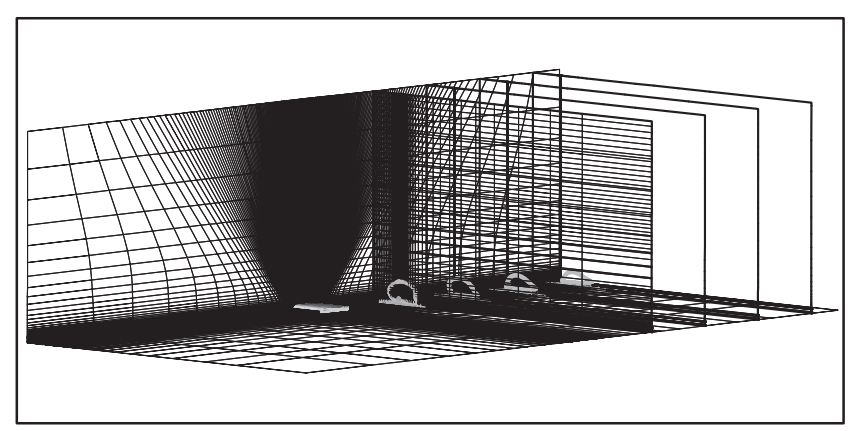

Fig. 6. Cutting planes.

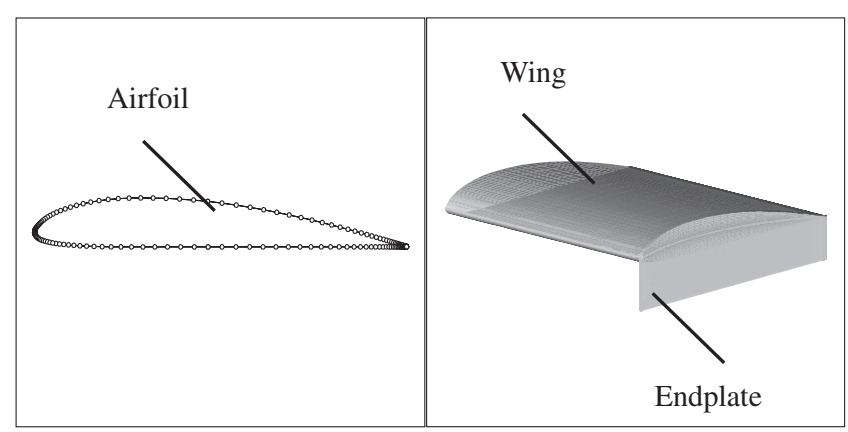

(a) Airfoil

(b) Wing and endplate

Fig. 7. Clark-Y airfoil and wing-endplate combination.

\subsection{Wing and endplate selection}

In general, the airfoil with DHMTU concept is used for the WIG. This airfoil is characterized by a flat low surface and an s-shaped camber line. These prevent a negative ground effect and increase longitudinal stability. Therefore, Clark-Y (Fig. 7(a)); which has the maximum thickness-tochord ratio of $12 \%$, was selected. ${ }^{15)}$

Another major feature of the WIG is the low-aspect-ratio wing; the wing span is restricted by the ground clearance so the total lift is a function of the chord length and height above the surface. Therefore, a rectangular wing with an AR of 2 is considered.

Low-aspect-ratio wings incur a pressure loss because of the flow dominance of wing-tip vortices; therefore, endplates are frequently included. To reduce pressure loss, the endplates protrude below the wing's lower surface as shown in Fig. 7(b). ${ }^{21)}$ Endplates are generated with depth ratios 


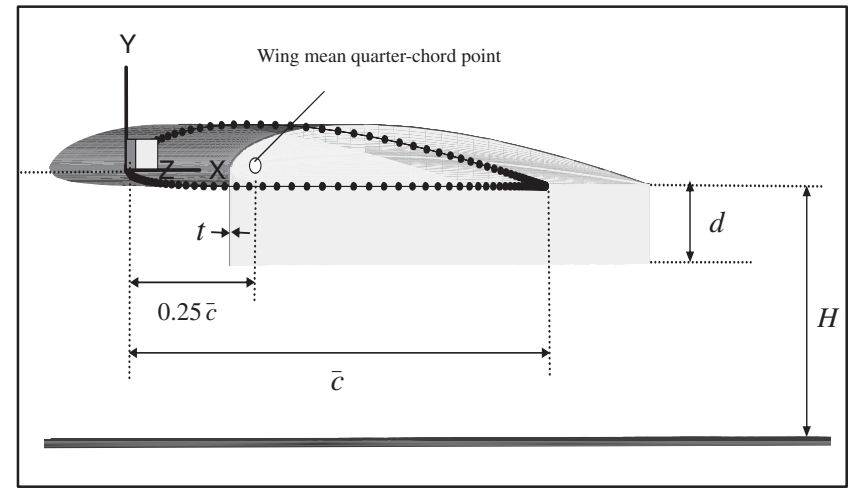

Fig. 8. Geometry of wing-endplate combination.

$(d / \bar{c})$ of 0.1 (WEP01) and 0.15 (WEP015). WEP is an abbreviation for With End Plate. Endplates are generated by extending the length as much as the depth ratio from the chord line. The origin of the height is a wing mean quarter-chord point. For example, at height ratio $(H / \bar{c}) 0.3$, the distance between the ground and wing mean quarter-chord point is $30 \%$ of the chord length. The thickness ratio $(t / \bar{c})$ of the endplate is $000625 .{ }^{22)}$ Figure 8 shows the geometry of the wing-endplate combination.

\subsection{Code validation}

This study compared two different drag prediction methods. Hence, the computation result must be validated first. The validation flow condition is described in section, 4.1. An H-type structured grid is used to generate a grid for attack angles of $0^{\circ}, 2^{\circ}, 4^{\circ}$, and $6^{\circ}$ without the endplates in free flight. Figure 9 shows good agreement between numerical and wind-tunnel investigation. ${ }^{22)} \mathrm{Next}$, the wing-mounted endplates were generated with depth ratio of 0.1. An H-type structured grid was also used to generate a grid for attack angles of $0^{\circ}, 2^{\circ}, 4^{\circ}$, and $6^{\circ}$ in free flight. Figure 10 shows slightly worse agreement between the numerical and wind-tunnel experimental data ${ }^{22)}$ in the drag polar chart.

\section{Results and Discussion}

\subsection{Comparison between total drag by BSI and WSI with ground effect}

Body surface integration and wake surface integration were conducted independently to compare the total drag values using a Clark-Y wing without an endplate. The results of the total drag comparison between the two integration methods are shown in Table 1. The position of the wake analysis is one root chord aft of the wing because the viscous term $\tau_{x x}$ is ignored in the final wake integral formulation.

Although drag prediction based on the wake surface integration shows disparities of $-1.96 \%$ to $4.35 \%$, the difference between the two integrations would be clearer if complex geometry was used. ${ }^{6)}$

In this study, the total drags for both integrations are similar. Hence, considering induced drag as a fraction of total drag is reliable. Therefore, the induced drag component can be extracted and the value is also reliable.

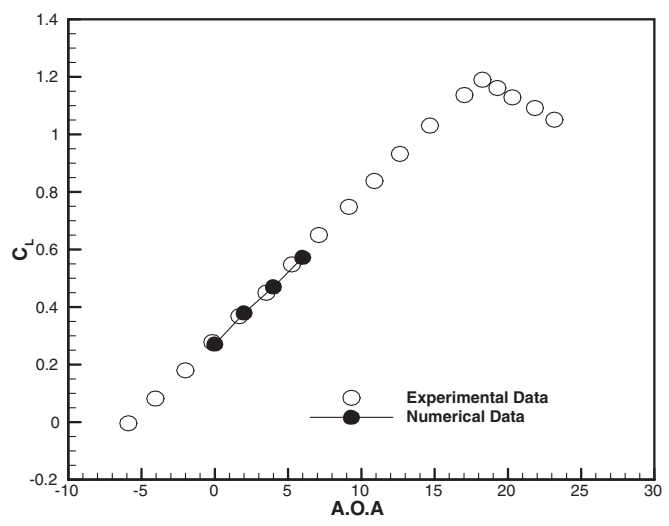

(a) Lift coefficient

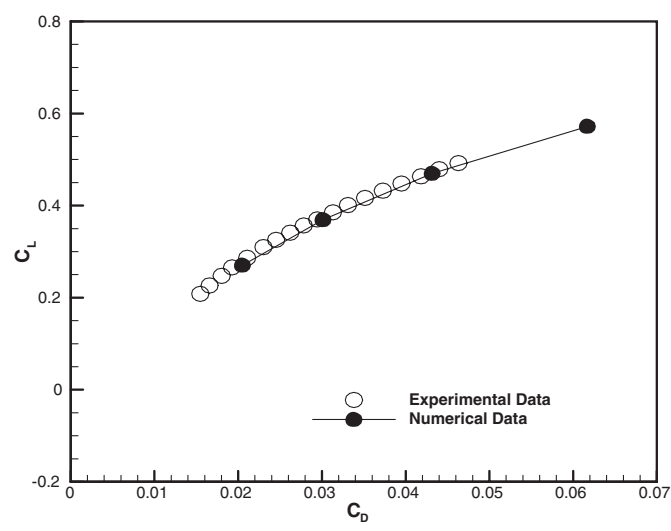

(b) Drag polar

Fig. 9. Code validation with Clark-Y wing.

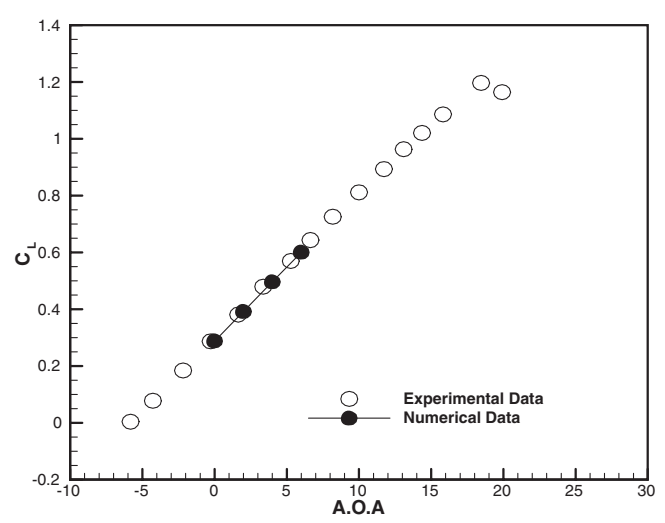

(a) Lift coefficient

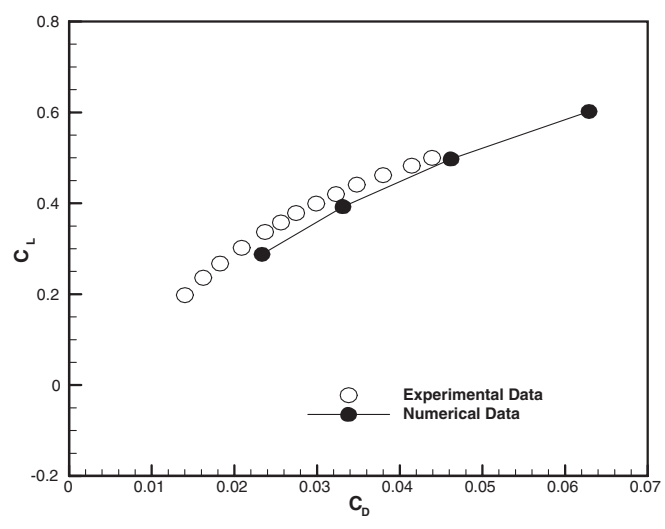

(b) Drag polar

Fig. 10. Code validation with wing-endplate combination. 
Table 1. Comparison between total drag by BSI and WSI.

\begin{tabular}{|c|c|c|c|c|}
\hline \multirow{2}{*}{ HR } & \multicolumn{4}{|c|}{ Drag coefficients } \\
\hline & \multicolumn{2}{|c|}{ BSI } & \multicolumn{2}{|c|}{ WSI } \\
\hline \multirow{4}{*}{0.7} & Pressure & 0.0161 & Induced & 0.0089 \\
\hline & Skin friction & 0.0043 & Entropy & 0.0111 \\
\hline & Total & 0.0204 & Total & 0.0200 \\
\hline & \multicolumn{3}{|c|}{ Total drag difference $(\%)$} & -1.96 \\
\hline \multirow{4}{*}{0.5} & Pressure & 0.0158 & Induced & 0.0085 \\
\hline & Skin friction & 0.0043 & Entropy & 0.0114 \\
\hline & Total & 0.0202 & Total & 0.0199 \\
\hline & \multicolumn{3}{|c|}{ Total drag difference $(\%)$} & -1.49 \\
\hline \multirow{4}{*}{0.3} & Pressure & 0.0149 & Induced & 0.0078 \\
\hline & Skin friction & 0.0043 & Entropy & 0.0114 \\
\hline & Total & 0.0193 & Total & 0.0192 \\
\hline & \multicolumn{3}{|c|}{ Total drag difference $(\%)$} & -0.52 \\
\hline \multirow{4}{*}{0.2} & Pressure & 0.0142 & Induced & 0.0068 \\
\hline & Skin friction & 0.0043 & Entropy & 0.0124 \\
\hline & Total & 0.0184 & Total & 0.0192 \\
\hline & \multicolumn{3}{|c|}{ Total drag difference $(\%)$} & 4.35 \\
\hline \multirow{4}{*}{0.1} & Pressure & 0.0122 & Induced & 0.0048 \\
\hline & Skin friction & 0.0042 & Entropy & 0.0121 \\
\hline & Total & 0.0165 & Total & 0.0169 \\
\hline & \multicolumn{3}{|c|}{ Total drag difference $(\%)$} & 2.42 \\
\hline
\end{tabular}

\subsection{Induced drag characteristics near ground}

The induced drag component was extracted at various heights. First, the induced drag in the ground effect was evaluated with a Clark-Y wing without an endplate (WOEP). As shown in Fig. 11, the induced drag in the ground effect evaluated by the wake surface integration differs from the induced drag value based on the lifting line theory (WOEP_Lifting line). However, the drop of the induced drag in the ground effect can be confirmed by Wieselsberger's theory (WOEP_Wieselsberger). ${ }^{23)}$ The result shows good agreement. As the wing approaches the ground, the induced drag is found to decrease near the ground, because wing tip vortices in free flight get weaker near the ground due to the restriction. Therefore, the increase in effective aspect ratio is obvious.

Figure 12 shows a remarkable discrepancy when the endplates (WEP01 and WEP015) are mounted on a wing tip. The gap between the lifting line theory (WEP01_ Lifting line and WEP015_Lifting line) and the calculated values increases because end plates prevent additional pressure loss producing wing-tip vortices. Moreover, Wiselsberger's theory (WEP01_Wieselsberger and WEP015_Wieselsberger) also overestimates induced drag very near the ground because this theory does not consider the contribution of the endplate to effective aspect ratio. $\left.{ }^{24}\right)$

From the computation, the wake surface integration satisfactorily shows the declining trend of the induced drag in the ground effect with a Clark-Y wing and wing-endplate combinations.

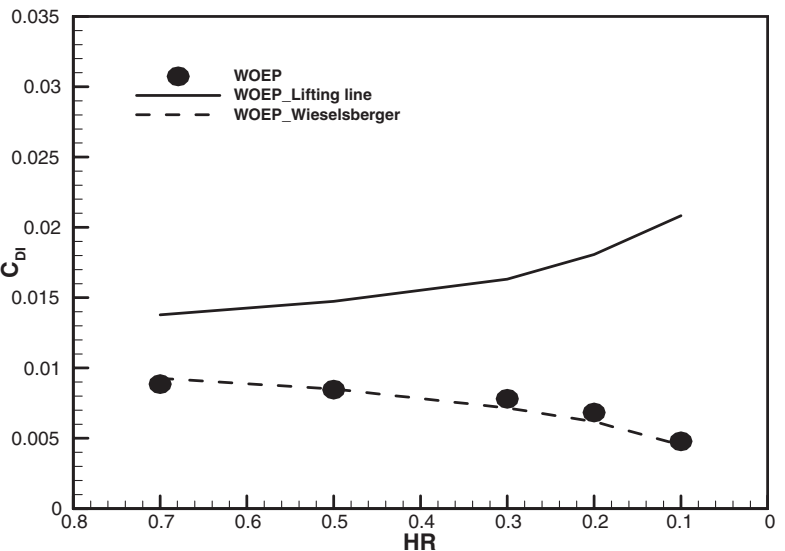

Fig. 11. Induced drag of wing in ground effect.

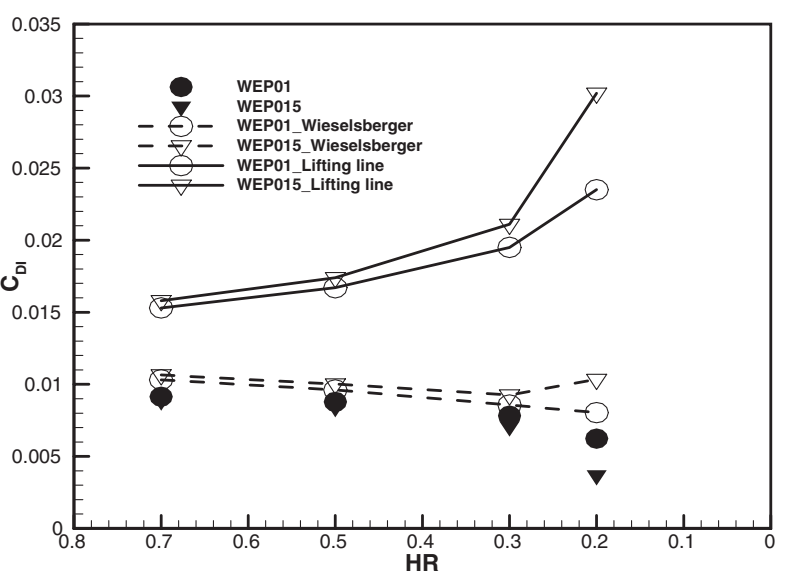

Fig. 12. Induced drag of wing-endplate in ground effect.

\section{Conclusion}

This study considered prediction of the induced drag in the ground effect using the wake surface integration method. Application of wake surface integration to cases of ground effect is valid because the moving boundary satisfies proposed assumption 4.

Drag prediction based on wake surface integration shows a disparity of $-1.96 \%$ to $4.35 \%$ compared to body surface integration. In this case, the total drags of the two integrations are similar, so considering the induced drag to be a fraction of the total drag is reliable. Therefore, the induced drag component can be extracted at various heights. Finally, induced drag in the ground effect was evaluated with Clark$\mathrm{Y}$ wing and Clark-Y wing-endplate combinations. The WIG has reduced induced drag in the ground effect due to the restriction of the ground. Moreover, induced drag further decreases with endplates because of prevention of additional pressure loss. From the computation, wake surface integration satisfactorily demonstrates the drop in induced drag in the ground effect with a Clark-Y wing and wing-endplate combinations. 


\section{References}

1) The WIG Page: http://www.se-technology.com/wig/index.php

2) Giles, M. B. and Cummings, R. M.: Wake Integration for ThreeDimensional Flowfield Computations: Theoretical Development, J. Aircraft, 36 (1999), pp. 357-365.

3) Yu, N. J., Chen, H. C., Samant, S. S. and Rubbert, P. E.: Inviscid Drag Calculations for Transonic Flows, AIAA Paper 83-1928, 1983.

4) Lock, R. C.: The Prediction of the Drag of Aerofoils and Wings at High Subsonic Speeds, Aeronautical J., 90 (1986), pp. 207-226.

5) Van Dam, C. P., Nikfetrat, K.,Wong, K. and Vijgen, P. M. H. W.: Drag Prediction at Subsonic and Transonic Speeds Using Euler Methods, J. Aircraft, 32 (1995), pp. 839-845.

6) Hunt, D. L., Giles, M. B. and Cummings, R. M.: Wake Integration for Three-Dimensional Flowfield Computations, Application, J. Aircraft, 36 (1999), pp. 366-373.

7) Chao, D. D. and van Dam, C. P.: Airfoil Drag Prediction and Decomposition, J. Aircraft, 36 (1999), pp. 675-681.

8) Chao, D. D. and van Dam, C. P.: Wing Drag Prediction and Decomposition, J. Aircraft, 43 (2006), pp. 82-90.

9) Van Dam, C. P.: Recent Experience with Different Methods of Drag Prediction, Progr. Aerospace Sci., 35 (1999), pp. 751-798.

10) Schmitt, V. and Destarac, D.: Recent Progress in Drag Prediction and Reduction for Civil Transport Aircraft at ONERA, AIAA Paper 980137, 1998.

11) Paparone, L. and Tognaccini, R.: Computational Fluid DynamicsBased Drag Prediction and Decomposition, AIAA J., 41 (2003), pp. 1647-1657.

12) Yamazaki, W., Matsushima, K. and Nakahashi, K.: Application of
Drag Decomposition Method to CFD Computational Results, AIAA Paper 05-4723, 2005.

13) Barber, T.: Aerodynamic Ground Effect: A case Study of the Integration of CFD and Experiment, Int. J. Vehicle Design, 40 (2006), pp. 299-316.

14) Chun, H. H. and Chang, R. H.: Turbulence Flow Simulation for Wings in Ground Effect with Two Ground Conditions: Fixed and Moving Ground, Int. J. Maritime Engineering, 145 (2003), pp. 51-68.

15) Zimmerman, C. H.: Characteristics of Clark-Y Airfoils of Small Aspect Ratios, NACA Report 431, 1933.

16) Shin, S. Y.: Study on Aerodynamic Characteristics of Wing in Ground Effect, Masters Dissertation, KAIST 2006.

17) Moore, N., Wilson, P. A. and Peter, A. J.: An Investigation into Wing in Ground Effect Aerofoil Geometry, RTO-MP-095, 2002.

18) Park, S. H.: Prediction Methods of Dynamic Stability Derivative Using the Navier-Stokes Equations, Doctoral Dissertation, KAIST 2003.

19) Park, S. H. and Kwon, J. H.: A Preconditioned HLLE Method for Flows at All Mach Numbers, AIAA Paper 04-2709, 2004.

20) Park, S. H. and Kwon, J. H.: Implementation of $k$ - $\omega$ Turbulence Models in an Implicit Multigrid Method, AIAA J., 42 (2004), pp. 1348-1357.

21) Handler, E. H.: Practical Considerations Regarding Wing-in-Ground Effect Aircraft, AIAA Paper 76-878, 1976.

22) Hinshaw, W. D., Sickies, W. E. and Hewin, L. M.: Wind-Tunnel Investigation of Ground Effect, Lockheed Report 16906, 1964.

23) Wieselsberger, C.: Wing Resistance near the Ground, NACA Report 77, 1922.

24) Reid, E. G.: A full-Scale Investigation of Ground Effect, NACA Report 263, 1926. 